\title{
WIDE BAND FREE SPACE TRANSMISSION LINK UTILIZING A MODULATED INFRARED LASER
}

\author{
Ralph J. Pasquinelli, Fermilab*, Batavia, IL
}

\section{Abstract}

For years, Fermilab has utilized wide bandwidth transmission links for stochastic cooling feed back systems. ${ }^{1,2,3}$ Stochastic cooling requires the feed back signal arrive at the kicker in time synchrony with the beam on the same turn. While coaxial cables suffice at lower microwave frequencies, their high loss and dispersion make them unsuitable at higher frequencies. Coaxial cables up to 125 meters in length have been utilized in the Antiproton Source. With the advent of the Recycler ring, (600-900 meters in diameter) coaxial cables have unacceptable losses. Stochastic cooling will be utilized in the Recycler to maintain a small beam emittance and momentum spread. A new means of transmitting the signal across the ring is necessary. While optical fibers have very low loss and excellent bandwidth characteristics, their propagation velocity is too slow to meet the beam on the other side of the ring. A modulated infrared laser is expanded from single mode fiber (utilizing telescopes) and transmitted in an evacuated pipe across the ring. The system will be described with preliminary results presented.

\section{ACCUMULATOR SYSTEM}

The first free space light link to be constructed at Fermilab was for the core transverse cooling systems in the Accumulator. These systems operate between 4-8 $\mathrm{GHz}$ with cable lengths approaching 100 meters. The insertion loss on half-inch coax is $18 \mathrm{~dB}$ to $28 \mathrm{~dB}$ for this length and frequency range. In addition, up to 360 degrees of phase dispersion is experienced, requiring a specialized phase equalizer.

These optical links provide flat amplitude and phase response for broadband microwave links. Typical response is plus minus $1 \mathrm{~dB}$ and 10 degrees phase flatness for the bandwidths mentioned. This comes at the expense of an overall insertion loss approaching $35 \mathrm{~dB}$.

A means of transmitting the signal in free space is required to meet the timing needs of stochastic cooling. An optical setup consisting of a fiber to graded index (GRIN) lens and a twenty-time telescope expander is utilized to enlarge the nine-micron diameter laser beam to approximately one-centimeter diameter. At

*Operated by Universities Research Association for the Department of Energy this size, the beam can propagate with minimal increase in spot size for the 76 meters of the accumulator test. A similar telescope is used on the receiving end to focus the 1310 nanometer beam onto a Hamamatsu fast photo diode. ${ }^{4}$ This photo diode not only has a response beyond $8 \mathrm{GHz}$, but also a large active area of 200 microns square. This large active area allows building a system that is relatively insensitive to random laser beam motion. (Earlier tests utilized a GRIN lens on the receiving end that required motion feedback.)

Because timing stability is critical to stochastic cooling performance, the transmission medium must have a stable propagation velocity. The velocity of light in a medium is inversely proportional to the index of refraction of the medium. In addition, the defocusing caused by changes in the index of refraction could cause the laser beam position to vary as a function of time. Anyone who has experienced "heat ripple" in a parking lot on a hot day has observed this phenomena. The only way to preserve the five picosecond timing tolerance is to transmit the laser through a vacuum.

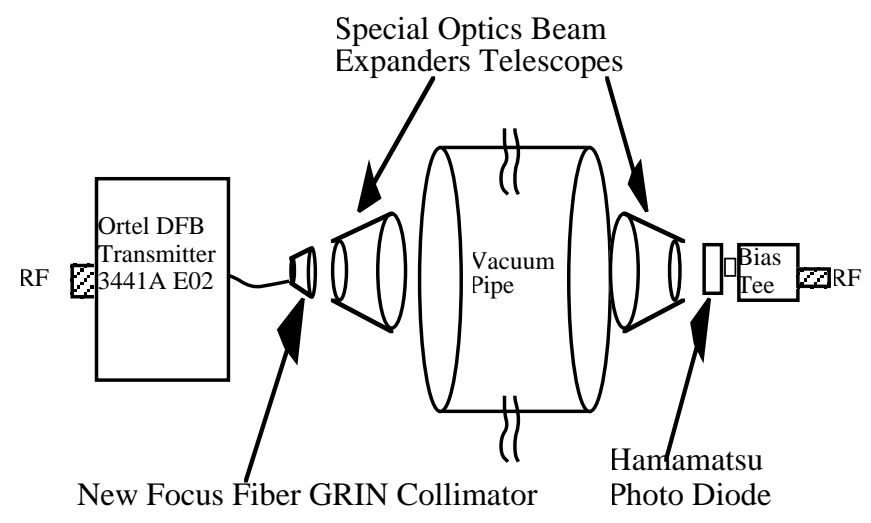

Figure 1. Optical link hardware configuration.

A twenty-inch diameter steel pipe was buried across a cord of the Accumulator ring to provide the light path for the laser beam. A Roots blower vacuum pump is utilized to reach a vacuum of approximately one Torr. Quartz vacuum windows provide the seal between the telescopes and the vacuum pipe. Figure 1 is a schematic of the test setup. 


\section{RECYCLER SYSTEM}

The Recycler Ring is the new antiproton depository storage ring that is being commissioned at Fermilab. ${ }^{5}$ This ring is fabricated with permanent magnets and is housed in the same enclosure with the Main Injector. Although electron cooling is planned for the future, stochastic cooling will be utilized initially. The size of the Recycler is approximately 3.3 kilometers in circumference. As with all stochastic cooling, feedback must be applied on the same turn of the beam for optimum performance. In the Recycler, this means a chord across the ring approaches 600 meters.
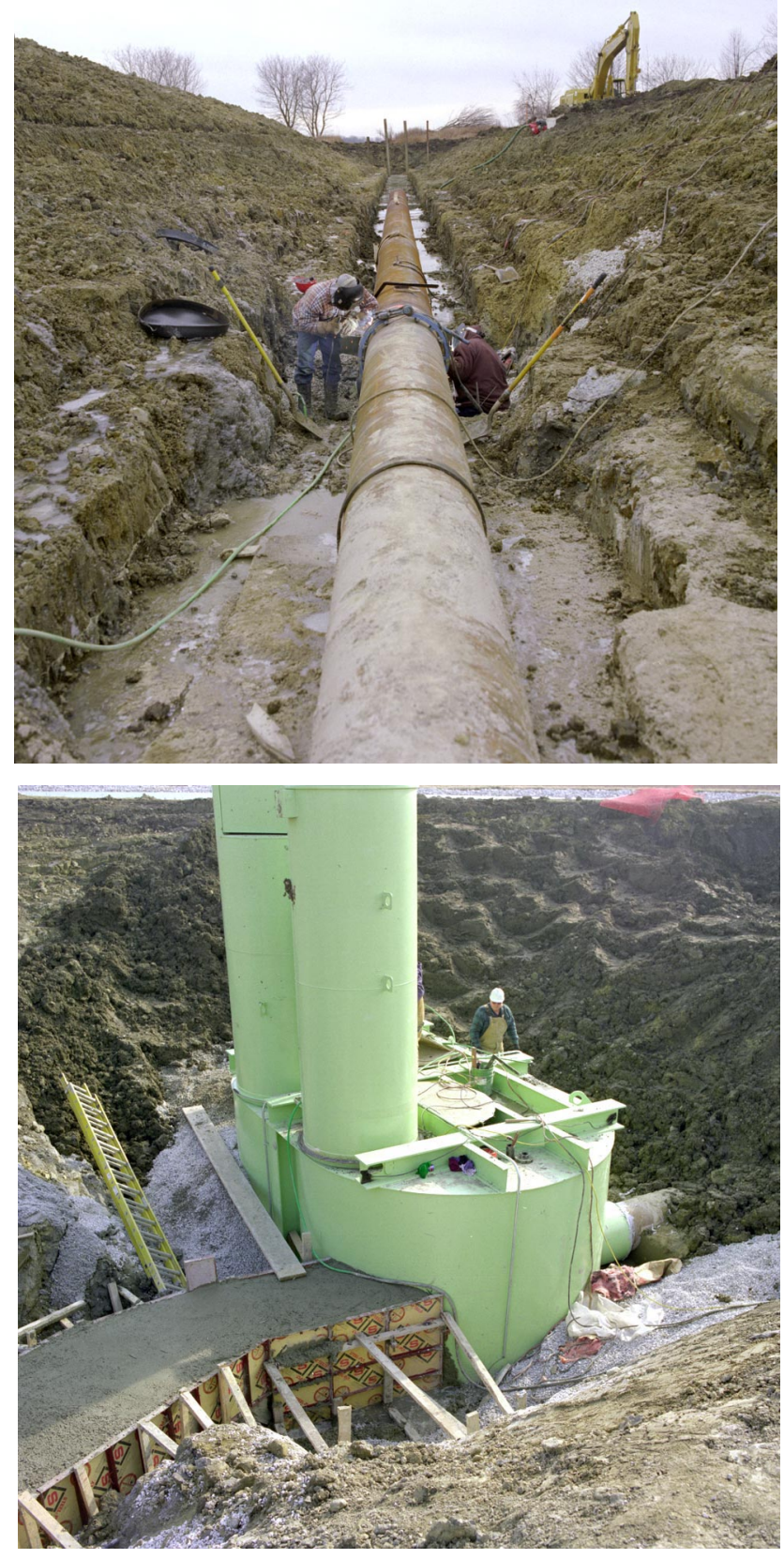

Figure 2. Top: light pipe installation. Bottom: Underground equipment enclosure, fondly referred to as the "peanut".
The cooling systems in the Recycler operate in the frequency bands of $0.5-4 \mathrm{GHz}$. Coax cable with a 7/8inch diameter would provide $20-60 \mathrm{~dB}$ insertion loss and phase dispersion of hundreds of degrees, both unacceptable. Even the best cable has a propagation velocity of $88 \% \mathrm{c}$, requiring a longer chord than a light link.

A civil construction project is currently underway to bury a 24-inch diameter steel pipe between the ten and twenty sectors of the Main Injector/Recycler complex. (Figure 2) There are a total of four cooling systems that will have their signals transmitted through this pipe. The large pipe size was chosen to minimize civil construction tolerances based on a trade off in material versus labor costs. At the ends of pipe, underground enclosures are buried just outside the minimum shielding requirements for the Main Injector, hence allowing unlimited occupancy. Beneficial occupancy of the light link enclosures is expected by April 1999. Initial light link tests will begin shortly there after. Commissioning of Recycler stochastic cooling is expected for later in the summer of 1999.

Due to the longer length of the Recycler light link, the laser beam will experience more beam dispersion. It is expected that the beam diameter will increase to near 8 centimeters. This coupled with a long baseline will add to the position sensitivity of the system. Bench experiments have shown that the laser beam can be focused to a full width of 100 microns utilizing the twenty-power telescope. Due to the large size of the photo detector (200x200 microns), it is hoped that position sensitivity will not be a problem.

\section{ACCUMULATOR PERFORMANCE}

A complete core vertical cooling system was installed in the Accumulator ring in July of 1997 before the long site shutdown to finalize Main Injector installation. This system was installed in parallel to the original cable based cooling system so that a side by side comparison could be made. Figure 3 shows the amplitude and phase transfer functions for the two systems under identical beam conditions. As can be seen from the cable based system, there is a high frequency degradation in signal to noise ratio because of the gain slope losses. The light link with its flat response shows improved signal to noise at the high frequency end of the band. The remnant gain slope present in both responses is due to bandwidth limitations of the system pickups and kickers. Figure 4 depicts the difference in signal suppression between the cable and light link system. A customized equalizer has been designed to remove the gain slope and will be installed in the near future. Both core transverse-cooling systems will be upgraded to light links for the startup of the Accumulator ring. 

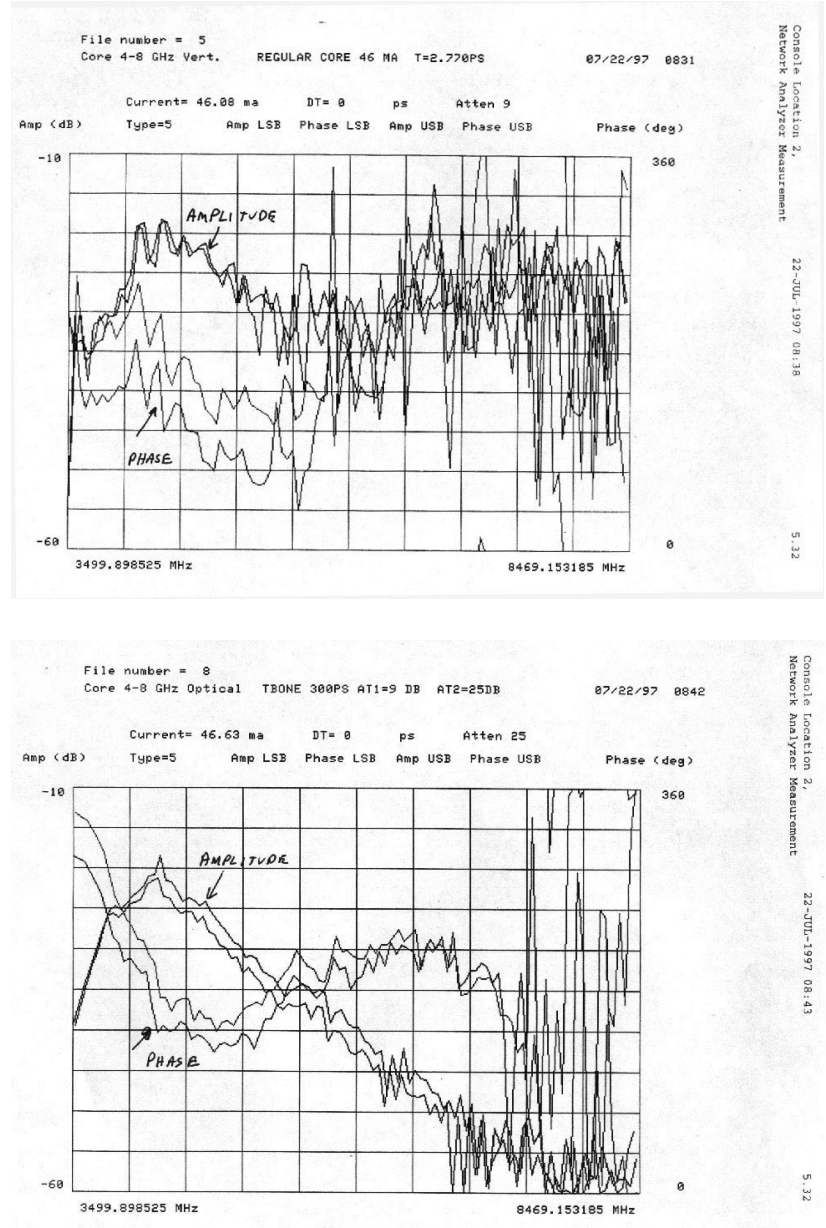

Figure 3. Accumulator beam transfer functions. Top: original cable transmission across ring. Bottom: optical link transmission across ring. Taken under identical conditions. Scale: $5 \mathrm{~dB}$ and 36 degrees per division. Frequency sweep: $3.5-8.5 \mathrm{GHz}$

\section{ACKNOWLEDGEMENTS}

The work to create the light links has been possible by the efforts of the Beams and FESS Divisions of Fermilab. The civil construction is critical to system performance. The diligence of the Antiproton Source Stochastic Cooling group made the Accumulator test possible.
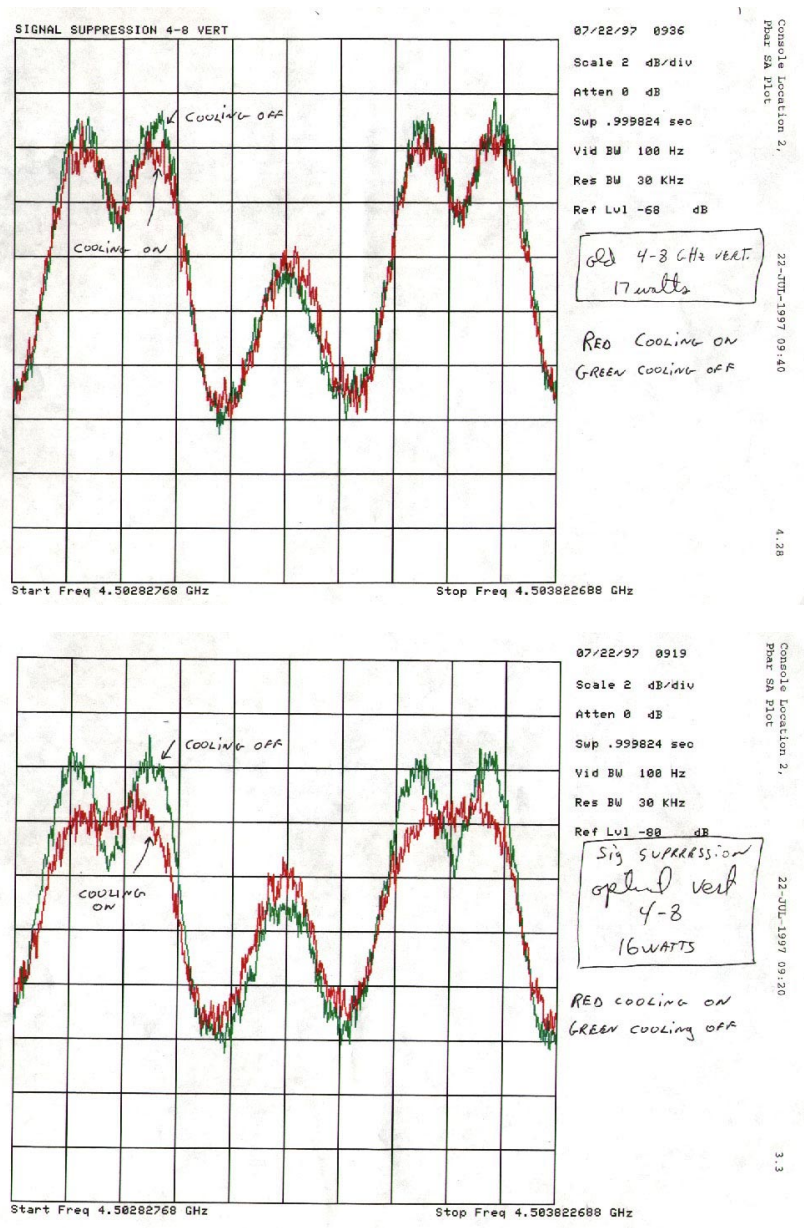

Figure 4. Signal Suppression measurements on Accumulator Beam. Top: Original cable transmission. Bottom: Optical link transmission. Red closed loop, Green open loop. Taken under identical conditions. Scale: $2 \mathrm{~dB} / \mathrm{div}$, Span $100 \mathrm{kHz}$ at $4.5 \mathrm{GHz}$

\section{REFERENCES}

[1] Electro-Optical Technology Applied to Accelerator Beam Measurement and Control, Ralph J. Pasquinelli, IEEE Nuclear Science, May 1993, Washington D.C.

[2] Fiber Optic Links for Instrumentation, Ralph J. Pasquinelli, Conference Proceedings of the Accelerator Instrumentation Workshop, October 1990

[3] Optical Notch Filters for Fermilab Debuncher Betatron Stochastic Cooling, Ralph J. Pasquinelli et. al., IEEE Nuclear Science, March 1989

[4] Hamamatsu Corporation, Ultrafast InGaAs MSM Photodetectors G7096 series, July 1997.

[5] Recycler Ring Technical Design Report; Gerry Jackson, editor, Fermilab internal report TM-1991, 1996. 